\title{
L'architecture comme expérience écologique de l'être au monde : le lieu du lien
}

\section{Olivier Lehmans}

\author{
(2) OpenEdition \\ Journals \\ Édition électronique \\ URL : http://journals.openedition.org/ere/1011 \\ DOI : $10.4000 /$ ere. 1011 \\ ISSN : 2561-2271 \\ Éditeur \\ Centr'ERE
}

Référence électronique

Olivier Lehmans, "L'architecture comme expérience écologique de l'être au monde : le lieu du lien », Éducation relative à l'environnement [En ligne], Volume 10 | 2012, mis en ligne le 20 décembre 2012, consulté le 21 février 2020. URL : http://journals.openedition.org/ere/1011 ; DOI : 10.4000/ere.1011 


\title{
L'architecture comme expérience écologique de l'être au monde : le lieu du lien
}

\author{
Olivier Lehmans
}

1 Pensée, construite, perçue, vécue, l'architecture exprime un rapport raisonné de l'homme à son environnement, rapport au « génie du lieu » (Norberg-Schultz, 1997). Elle offre les moyens d'une appropriation de l'espace à travers un jeu formel entre les limites et les usages. Longtemps déconnectée des contraintes naturelles de l'environnement grâce aux progrès des matériaux et des techniques au vingtième siècle qui permettent pratiquement d'exclure du monde les usages du bâtiment en " conditionnant " l'air et l'espace et souvent mise en valeur dans ses dimensions spectaculaires et symboliques, l'architecture évolue aujourd'hui vers une réflexion renouvelée sur son rapport à l'environnement naturel et social.

2 Ce renouvellement se traduit dans la normalisation et la labellisation qui imposent aux architectes et aux professionnels du bâtiment une prise en compte de l'impact environnemental - essentiellement énergétique - de la construction (haute qualité environnementale, $\mathrm{HQE}$, bâtiment basse consommation, $\mathrm{BBC}$ ), et plus généralement dans l'évolution d'une partie de la profession de plus en plus attentive aux dimensions écologiques de l'architecture à travers, notamment, l'éco-conception. Cette évolution tend à rappeler que l'architecture, comme l'urbanisme et le paysage, relie les hommes au territoire, ou plus précisément à l'œekoumène au sens précisé par Augustin Berque (2000, p. 12-13) de "relation à la fois écologique, technique et symbolique de l'humanité à l'étendue terrestre ». Cette relation aux lieux est ontologique et sociale, elle est créatrice de lien. L'architecture est le lieu du lien au moyen de résolutions techniques, le «lieu apprenant» (Schaller, 2007). L'habiter qu'elle appelle correspond ainsi à un " être au monde », une expérience intime et partagée de l'environnement à travers les limites des espaces, les sensations des matériaux et des ambiances.

3 Cependant, les pratiques de construction imposées par un marché mondialisé et des industries très puissantes, ainsi que les stéréotypes sociaux, politiques et esthétiques 
concernant l'habitat n'évoluent que très lentement. C'est pourquoi il est essentiel que les architectes aient une démarche pédagogique ouverte et partagent leur réflexion en sensibilisant le public aux enjeux environnementaux de l'espace construit et habité. Deux exemples de ce travail de sensibilisation sont proposés pour traduire le processus de mise au monde de l'habiter dans la matérialité de l'architecture comme projet d'expérience vécue, intime et sociale, réelle et imaginaire, naturelle et culturelle. Dans ces deux exemples, l'architecte est un médiateur. On reprend ici la fonction essentielle que Jean Caune (2010, p. 97) assigne à la médiation: «(..) mettre l'accent sur la relation: entre les personnes, entre les institutions, entre les langages, entre les formes " grâce à l'énonciation qui se réalise dans l'acte créateur. Le projet "Cabanes, construis ton aventure" fait de l'espace construit un outil de médiation vers des apprentissages transversaux liés à la perception par les enfants de l'environnement, tandis que le projet de Maison éco-citoyenne interpelle les citoyens et appelle leur réflexion et leur engagement sur leur responsabilité dans l'évolution des espaces habités.

\section{Cabanes : l'architecture comme médiation pédagogique}

4 Le projet «Cabanes » s'est fait dans le cadre d'un projet d'action culturelle (PAC) géré par le ministère de l'Éducation nationale et l'Institut français d'architecture, à l'époque où l'action culturelle disposait encore de quelques moyens. Il a été mené en 2002 par une équipe constituée d'enseignants de l'école Paul Bert à Bordeaux, d'une paysagiste, Anne-Gaël Le Guillanton et de l'architecte. L'objectif du projet était de faire participer les élèves et les enseignants à la maitrise d'ouvrage d'une cabane permettant la communication entre les écoles maternelle et élémentaire séparées, mais mitoyennes tout en les sensibilisant aux dimensions environnementales de la façon d'occuper l'espace.

\section{Objectifs}

5 Fruit d'un travail commun entre les élèves de grande section de maternelle et de cours préparatoire, le projet visait à créer du lien, à marquer la continuité spatiale et celle des apprentissages des élèves entre cinq et sept ans. Projection vers un ailleurs pour les plus petits, réflexion introspective pour les plus grands, espace de liberté dans le fonctionnement contraint de l'école, point de contemplation et d'expérimentation du paysage, la cabane est un lieu de transfert et un dispositif de culture collective.

6 La conception de la cabane avec les élèves et les enseignants a été aussi importante que sa construction. Sous ses modestes proportions, la cabane a l'avantage de concentrer la plupart des questionnements essentiels de l'architecture, perçus spontanément par les enfants quand on les y incite. L'intérêt de ce projet réside surtout dans l'initiation d'une pensée architecturale, fruit de l'apprentissage de la société et de la culture de la cité. Les élèves et les enseignants font l'expérience de "la maîtrise d'ouvrage ", l'architecte restant le maître d'œuvre. De ce fait, les élèves et les enseignants sont les acteurs du processus de création de la cabane avant d'en être les utilisateurs. La médiation se fait essentiellement dans le dialogue qui se noue et la réflexion qui 
s'engage entre les élèves, l'enseignant et les architectes sur le sens de l'acte de construire.

\section{Démarche}

7 Ce travail a nécessité plusieurs étapes. Dans une première phase d'observation et de pré-programmation, sont mises en place la réflexion et la verbalisation sur les représentations réciproques (perceptions de l'espace: limites, frontières, usages, rencontres, matières, couleurs, lumières, sons, histoires vécues des frères et sœurs, etc.), l'analyse de l'utilisation de l'espace de la classe (différences et ressemblances entre la grande section et le cours préparatoire), les repérages (photos, notes et dessins faits par les enfants, les enseignants, les architectes). Dans une seconde phase de programmation du projet, le site envisagé est observé (à l'intersection de la classe de grande section et de la cour de l'école élémentaire), un relevé d'état des lieux (apprentissage de la lecture des plans) et un diagnostic (mots, dessins, images rapportées) réalisés. Dans une troisième phase de présentation du projet par les architectes, maîtres d'œuvre, les plans, les dessins, les images, la maquette réalisés par les professionnels font l'objet d'un recueil des impressions et des questionnements des élèves et des enseignants, et permettent une analyse de la perception collective du projet.

8 Ces phases de mise en projet sont essentielles dans le processus de médiation qui suppose une réflexion commune. Par la suite, la médiation se fait surtout à travers l'accompagnement des usages et des représentations de l'espace. Dans la phase de réalisation du projet, les étapes de la construction et de la formalisation sont clairement définies : ouverture du chantier, panneau d'information de chantier, suivi des travaux, reportage mené par les élèves (photos, échantillonnage d'objets utilisés pour les travaux, matériaux et outils, vocabulaire lié à la construction, rencontre éventuelle avec l'artisan). La réception de l'ouvrage fait l'objet d'un évènement avec l'inauguration, la fête, la mise en service (observations, commentaires, présentation du journal du projet). La phase d'appropriation permet l'utilisation réelle de l'espace et les découvertes de ses qualités à travers les usages guidés par les adultes. La mise en place d'outils de communication (écriture, signes, images, plantations) autour de, et dans la cabane achève de construire un espace apprenant et permet d'analyser les transformations acquises. Enfin, une dernière phase de démontage et de recyclage laisse l'espace libre pour de nouvelles découvertes.

\section{Évaluation}

9 L'expérience de la cabane a permis de croiser dans la démarche pédagogique une réflexion sur la relation entre lien social, lien cognitif et lien spatial, et sur l'ancrage de la forme habitée dans un territoire. Il a en effet été choisi de construire les cabanes dans un matériau local, les palettes de bois en pin maritime des Landes, qui, à la fin du projet, ont été restituées et réutilisées. Les palettes sont des éléments modulaires préfabriqués issus de l'industrie, qui ont été utilisés sans bricolage afin de préserver le lien à l'environnement régional et à l'économie locale. L'industriel a été directement associé au projet, et les élèves sensibilisés au fait qu'il est important de privilégier une 
production locale ne nécessitant que peu de transport et favorisant une économie durable.

Tous les jours, les enfants ont pris rendez-vous dans les cabanes pour se retrouver, se détendre, lire des histoires. L'expérience a été très positivement vécue bien qu'éphémère. Le principe même du recyclage imposait la disparition de l'objet une fois sa fonction remplie : créer du lien, se penser dans l'environnement.

11 Les contraintes scolaires liées à la sécurité, à la gestion du temps et de l'espace, aux préoccupations pédagogiques des enseignants qui ne rejoignent pas spontanément les objectifs d'éducation à l'environnement et à l'architecture ont posé des limites certaines à l'expérience par rapport aux fonctions de la cabane telles que les décrit Dominique Bachelart (2010). L'exigence de liberté, de découverte autonome, le droit à la régression n'ont pas toujours leur place à l'école. Les cabanes ont cependant permis d'ouvrir des horizons possibles. C'est au contraire, et à une échelle très différente, une expérience pérenne que la Maison éco-citoyenne de Bordeaux permet de vivre, toujours dans la logique d'un lieu apprenant.

\section{Maison éco-citoyenne : l'architecture comme accompagnement culturel et politique}

12 La Ville de Bordeaux a souhaité réaliser dans des locaux mis à disposition par la Communauté urbaine de Bordeaux (CUB), et situés à un point stratégique des quais, un lieu exemplaire d'information et d'apprentissage de l'écologie urbaine. La Maison écocitoyenne est ouverte au grand public, mais aussi aux institutions éducatives, aux associations et aux professionnels, pour leur permettre de comprendre les enjeux du développement durable dans la construction et les modes de vie.

\section{Objectifs}

13 C'est dans une double perspective symbolique et pédagogique que l'équipe de conception a travaillé pour un projet qui ne joue pas uniquement sur les images et un environnementalisme de façade, afin de construire une véritable réflexion dans la mise en œuvre de techniques innovantes en ciblant plusieurs objectifs. La mise en scène de l'œuvre architecturale dans son cadre, en premier lieu, est valorisée. La Maison écocitoyenne est le produit d'une réflexion globale sur la problématique de la construction durable dans la ville, notamment dans le cadre de la réhabilitation des bâtiments anciens pour leur attribuer des qualités environnementales, une mise en œuvre contemporaine de la construction en continuité avec les techniques et les matériaux du patrimoine local, dans la démarche HQE et la maîtrise des dépenses d'énergie, du coût global de construction et de développement durable.

En second lieu, l'image et le sens sont mis en relation à l'intérieur du bâtiment. Son volume conduit à imaginer un parcours initiatique intérieur organisé pour accueillir l'ensemble des fonctions et des espaces dans une logique d'expression de la nécessité d'une éco-citoyenneté. Les matières, les couleurs, l'apport de lumière naturelle, et les perspectives intérieures de la Maison véhiculent une sensation de bien-être et de sérénité, sans tomber dans l'imagerie passéiste et naïve de la maison écologique bricolée et brinquebalante. 
15 En troisième lieu, l'organisation des espaces s'articule autour des expositions, de l'accueil et des informations contenues dans le matériau même de la Maison. Celle-ci est un lieu d'exposition, mais aussi d'expérimentation, de documentation et de rencontre. La culture scientifique en est un des enjeux majeurs. La scénographie met donc en scène des parcours et des rencontres, une découverte intime et plurielle des enjeux de l'environnement. Ces parcours ne sont pas excluants puisque l'accent est mis sur l'accessibilité qui a permis au bâtiment d'être labellisé. Enfin, l'équipe d'architecture accompagne la maîtrise d'ouvrage sur la définition et la mise en œuvre des contenus pédagogiques en associant le réseau associatif et institutionnel lié aux questions environnementales (GRAINE, ADEME, Écocentres, etc.).

\section{Démarche}

La transformation du bâtiment est conçue en démarche de haute qualité environnementale (HQE). Le référentiel HQE implique un examen des procédés et matériaux entrant dans la construction en fonction de leurs propriétés selon une méthode d'analyse multicritères permettant de confronter pour chaque fonction les qualités architecturales, les performances techniques, les conditions de fabrication et de mise en œuvre, les effets sur l'environnement et la santé, les coûts directs et différés, la facilité d'entretien, de maintenance et de nettoyage. Même s'il est formel, il constitue un outil efficace de dialogue entre maîtrise d'œuvre et maîtrise d'ouvrage sur la réflexion environnementale.

Le projet applique un ensemble de principes constructifs et de techniques qui sont destinés à être habités, expérimentés et appropriés par le public :

- La reconquête de la biodiversité et la protection de l'environnement naturel : La végétalisation de la toiture est proche de la ripisylve avec des plantes résistantes, locales, nécessitant peu d'entretien. Elle accueille des ruches, des abris à papillon et des nichoirs à oiseaux.

- Une approche bioclimatique : L'espace central de la Maison éco-citoyenne bénéficie d'un éclairage naturel zénithal indirect et donc très confortable. La toiture végétalisée apporte de l'inertie au bâtiment et renforce le confort thermique. La ventilation naturelle minimise les consommations d'énergie. L'accent est mis sur les énergies renouvelables. Des panneaux solaires thermiques sont disposés en toiture pour les besoins en eau chaude sanitaire. Des capteurs solaires photovoltaïques souples sont intégrés à la couverture et permettent la revente d'électricité à ERDF. Une chaudière à bois sert au chauffage par le sol combiné avec le solaire.

- Une approche écologique : Dans la mesure du possible, et compte tenu des contraintes liées à l'accueil du public, les matériaux choisis sont sains (bois, chaux naturelle, isolation en fibre végétale) issus de filières courtes et biologiques. Les entreprises choisies sont locales et emploient du personnel en réinsertion.

\section{Évaluation}

Le travail de l'architecte s'arrête au moment de la réception du bâtiment, même s'il est souvent sollicité pour des visites ou des présentations, ce qui permet de poursuivre la médiation au-delà de la relation avec le maître d'ouvrage. Celui-ci a tendance à privilégier la médiatisation à court terme sur la médiation, qui se construit sur le long 
terme. Le caractère innovant et expérimental des techniques et des matériaux exige un effort d'adaptation et de réflexion de la part des utilisateurs qui ne s'impose pas facilement s'ils ne sont pas impliqués dans une démarche partagée et continuée. Ainsi, un an après son ouverture, la production d'électricité par les capteurs photovoltaïques n'était toujours pas rendue effective par un raccordement au réseau électrique. Le lien avec d'autres acteurs culturels (bibliothèque, centre de culture scientifique et technique) et éducatifs (établissements scolaires) restait ténu.

Le projet de Maison éco-citoyenne a été inauguré en octobre 2010. Il n'est donc pas encore possible d'en évaluer précisément l'impact du point de vue de la sensibilisation du public aux enjeux environnementaux de l'architecture et du bâti dans la ville. Il a provoqué un travail de réflexion et de concertation avec les services de collectivités territoriales concernées. Il donne forme au projet éducatif sur l'environnement autour des questions liées à l'Agenda 21 notamment. Il commence à mobiliser l'ensemble des acteurs du tissu associatif local.

\section{Conclusion}

Ces projets sont marginaux dans le paysage architectural qui ne valorise pas l'attention à l'ensemble des dimensions environnementales de la construction, mais reste centré sur des dimensions spectaculaires partiellement couvertes par la labellisation HQE ou un habillage vert qui manque parfois de cohérence. Ils pointent la responsabilité des architectes à la fois dans les transformations du paysage habité et leur impact sur l'environnement, et dans la sensibilisation du public à l'importance des enjeux environnementaux de la construction. Cette sensibilisation vise à donner aux habitants de la ville les moyens de devenir de véritables acteurs à la fois de la politique urbaine et de leur propre rapport culturel à l'espace construit. Les projets nous rappellent, avec Thierry Paquot (2007, p.16), qu'« habiter est une exigence de liberté, un devoir d'humanité, un combat incessant avec la barbarie ordinaire. Habiter s'apparente à une respiration onirique qui nous enveloppe et nous transporte. » Et seule la perspective écologique offre à l'homme cette possibilité imaginaire et créative.

\section{BIBLIOGRAPHIE}

Bachelart, D. (2010). Cabanes : aires de jeux libres et petits fragments de nature (artificielle) à ménager pour les enfants. Lettre du Graine, 19, 23-26.

Berque, A. (2000). Écoumène: Introduction à l'étude des milieux humains. Paris : Belin. Blanc, N. (2004). De l'écologique dans la ville. Ethnologie française, 2, 601-607.

Caune, J. (2010). Pratiques culturelles et médiation artistique : la construction du lien social. In Liquète, V. (coord.), Médiations. Les Essentiels d'Hermès (p. 85-102). Paris : CNRS Éditions.

Mancebo, F. (2007). Culture, marquage territorial et appropriation du développement durable. L'information géographique, 3, 79-87. 
Norberg-Schultz, C. (1997). Genius Loci. Paysage, ambiance, architecture (3e éd.). Paris : Mardage Éditions.

Paquot, T. (2007). Habiter, le propre de l'humain. Paris : La Découverte.

Schaller, J.-J. (2007). Un lieu apprenant : de l'habitus à l'historicité de l'action. L'orientation scolaire et professionnelle, 36(1), 83-93.

\section{AUTEUR}

\section{OLIVIER LEHMANS}

Architecte depuis 1992, après un séjour aux États-Unis, Olivier Lehmans a découvert le concept de sustainable architecture et suivi plusieurs formations sur l'écologie humaine. Il consacre toute son activité à l'architecture écologique et à l'éco-conception en France et à l'étranger. Il reste également très attaché aux fonctions pédagogiques des architectes et à leur responsabilité sociale et environnementale, tant au niveau national que dans les relations entre les pays et les cultures, notamment les plus fragiles. 